\title{
Three-dimensional numerical simulations of impulsively generated MHD waves in solar coronal loops
}

\author{
M. Selwa ${ }^{1}$, K. Murawski ${ }^{1}$, and G. Kowal ${ }^{2}$ \\ ${ }^{1}$ Institute of Physics, UMCS, ul. Radziszewskiego 10, 20-031 Lublin, Poland \\ e-mail: mselwa@kft.umcs.lublin.pl \\ 2 Astronomical Observatory, Jagiellonian University, ul. Orla 171, 30-244 Kraków, Poland
}

Received 21 January 2004 / Accepted 26 March 2004

\begin{abstract}
Impulsively generated magnetohydrodynamic waves in a typical EUV solar coronal loop are studied numerically using a three-dimensional FLASH code. Our results reveal several 3D effects such as distinctive time signatures which are collected at a detection point inside the loop. A slow magnetosonic wave generates a significant variation in the mass density profile with a time scale of the order of $40 \mathrm{~s}$. A fast kink wave affects the mass density, too, but its magnitude is much lower than in the case of a slow wave. Time scales which are associated with the fast kink wave are generally shorter than in the case of a slow wave; the former are in the range of a dozen or so seconds. Temporal signatures of a fast sausage wave reveal $\sim 5$-s oscillations in the quasi-periodic phase. Impulses which are launched outside the loop excite several-second oscillations in the mass density. Time signatures depend on the position of the detection point; they are usually more complex further away from the exciter.
\end{abstract}

Key words. waves - Sun: corona - Sun: oscillations

\section{Introduction}

This research has been motivated by the new observations of oscillations of coronal loops, detected by the spacecrafts TRACE (Handy et al. 1999) and SUMER/SOHO (Wilhelm), and in the ground-based solar eclipse imaging instrument SECIS (Phillips 2000; Aschwanden et al. 1999; Aschwanden 2003; Nakariakov 2003). Among them there are short period (1-10 s) oscillations which have been observed for several decades in the modulated radio emission (e.g., Aschwanden 2003), but only very recently have been observationally identified in the optical band as propagating waves in a coronal loop (Williams et al. 2002). These oscillations are only in SECIS data, TRACE and SOHO don't have this resolution. It is believed that these oscillations are associated with MHD modes of coronal plasma structures and, consequently, are an ideal tool for coronal seismology (Nakariakov et al. 1999; Nakariakov \& Ofman 2001; Nakariakov et al. 2004), an idea first put forward by Roberts et al. (1984). An understanding of these oscillations is particularly important because they may shed some light on the puzzles of coronal heating and the solar wind acceleration, providing seismic information about the coronal plasma.

The theory of coronal loop oscillations has been developed in some detail for the special case of a straight and infinite cylinder of magnetic field by Edwin \& Roberts (1983) who showed that MHD waves are guided by regions of low Alfvén speed, typically corresponding to regions of high mass density, and so are able to form distinctive wave packets. Such regions provide wave guides for fast magnetoacoustic waves. Slow and Alfvén waves are naturally guided along magnetic field lines. In fact the knowledge of MHD waves in the solar corona has increased significantly over the last few years. For instance, an extensive investigation of MHD waves in coronal loops has been carried on (e.g., Murawski \& Roberts 1994; Murawski et al. 1998; Nakariakov et al. 2004). In particular, Nakariakov et al. (2004) extended the model of Murawski \& Roberts (1994) to the developed stage of the fast wave evolution and showed that the fast magnetosonic waves exhibit a characteristic "tadpole" wavelet spectrum. However, the improved resolution of modern observational techniques motivates the further development in the study of theoretical aspects of coronal wave activity (Aschwanden 2003).

A number of theoretical aspects of the study of coronal loop oscillations, connected with the effects of nonlinearity, stratification and 3D structuring remain to be revealed. This paper is devoted to current numerical modelling of MHD wave processes.

As a consequence of the complexity of MHD waves in a highly inhomogeneous coronal plasma it is necessary to understand simpler phenomena which may play the role of elementary building blocks in the construction of a more elaborate theory. As a result, our strategy is to develop simpler models at the initial stage of the research and progressively extend 
and generalize them to more complex models at subsequent stages. The three-dimensional simulations we propose in these studies are motivated by the fact that real coronal loops in fact exhibit a multi-dimensional geometry. Traditionally, the study of kink and sausage modes of a straight magnetic cylinder are performed in the $2.5 \mathrm{D}$ geometry. This approach allows us to disregard both the linear coupling of various modes and model processes of excitation of the modes by an external asymmetric source. Thus, in this study, we model MHD oscillations of coronal loops with the use of a full-MHD 3D numerical code. Additionally, the 3D simulations allow us to study effects of excitation.

This paper concerns an infinitely long cylinder of a circular cross-section, and time signatures which are made by a wave signal measured at a fixed spatial location. The results of numerical simulations will be compared with the earlier results (Murawski \& Roberts 1994; Murawski et al. 1998) and with the observational data (Aschwanden 2003). MHD waves will be excited impulsively inside a coronal loop. In these parametric studies, the impulses will possess various spatial profiles. This study is relevant to the case when the wavelength is shorter than the scale height.

This paper is organized as follows. A simple model of coronal loops is developed in Sect. 2. In Section 3 we present a numerical model for loop waves. Section 4 contains results of the numerical simulations for impulsively generated MHD waves in a coronal loop. This paper is concluded by the presentation and discussion of the main results in Sect. 5.

\section{Physical model of impulsive waves in a coronal loop}

We consider the coronal plasma which is described by the following ideal magnetohydrodynamic equations:

$$
\begin{aligned}
& \frac{\partial \varrho}{\partial t}+\nabla \cdot(\varrho \boldsymbol{V})=0, \\
& \frac{\partial(\varrho \boldsymbol{V})}{\partial t}+(\varrho \boldsymbol{V} \cdot \nabla) \boldsymbol{V}=-\nabla p+\frac{1}{\mu}(\nabla \times \boldsymbol{B}) \times \boldsymbol{B}, \\
& \frac{\partial p}{\partial t}+\nabla \cdot(p \boldsymbol{V})=-p(\gamma-1) \nabla \cdot \boldsymbol{V}, \\
& \frac{\partial \boldsymbol{B}}{\partial t}=\nabla \times(\boldsymbol{V} \times \boldsymbol{B}), \\
& \nabla \cdot \boldsymbol{B}=0,
\end{aligned}
$$

where $\varrho$ is the mass density, $\boldsymbol{V}$ is the flow velocity, $\boldsymbol{B}$ is the magnetic field, $p$ is the pressure, $\mu$ is the magnetic permeability, and $\gamma=5 / 3$ is the adiabatic index.

As, in a realistic model of a coronal loop, a number of effects crowd in and complicate our understanding of wave phenomena we assume a simple coronal loop model. In this way we consider briefly some of the effects that require evaluation if we are to explain the observed wave signatures in coronal loops. We enquire as to what effects are most likely important, and estimate the time scales they produce.
In the model we examine a magnetically structured atmosphere in which the magnetic field is uniform and directed in the $z$-direction. We ignore the effects of gravity, field line curvature, twisted loops, non-circular cross-sections and we approximate the coronal loop by smooth plasma profiles in which the inhomogeneity occurs in the $r$-direction, and the $\phi$-direction is perpendicular both to the $r$ - and $z$-directions. In particular, we consider a loop of a cross-section radius $a$, field strength $B_{0}$ and mass density $\varrho_{0}$ embedded in a magnetic environment with field strength $B_{\mathrm{e}}$ and mass density $\varrho_{\mathrm{e}}$. The mass density $\varrho_{0}(r)$ profile is chosen as (Murawski et al. 1998)

$\varrho_{0}(r)=\varrho_{\mathrm{e}}+\left(\varrho_{\mathrm{i}}-\varrho_{\mathrm{e}}\right) \operatorname{sech}^{4}(r / a)$,

where $a$ is the loop radius, indices " $\mathrm{i}$ " and "e" denote quantities inside the loop and outside the loop, respectively. The equilibrium magnetic field $B_{0}(r) \hat{z}$ and pressure $p_{0}(r)$ profiles must satisfy the total pressure balance condition

$\frac{\mathrm{d}}{\mathrm{d} r}\left(p_{0}(r)+\frac{B_{0}^{2}(r)}{2 \mu}\right)=0$.

Hence, at the equilibrium the total (gas plus magnetic) pressure has to be constant. In particular, the total pressure inside the coronal loop is equal to the total pressure outside the coronal loop, i.e.

$p_{\mathrm{i}}+\frac{B_{\mathrm{i}}^{2}}{2 \mu}=p_{\mathrm{e}}+\frac{B_{\mathrm{e}}^{2}}{2 \mu}$.

We adopt the magnetic field profile which corresponds to an open magnetic structure, viz.

$B_{0}(r)=B_{\mathrm{e}}+\left(B_{\mathrm{i}}-B_{\mathrm{e}}\right) \operatorname{sech}^{4}(r / a)$

and the expression for $p_{0}(r)$ follows then from Eq. (7).

Similarly as in Murawski et al. (1998) we specify the plasma $\beta$, density ratio $d$, and the ratio of Alfvén speeds $v$ as

$\beta \equiv \frac{2 \mu p_{\mathrm{e}}}{B_{\mathrm{e}}^{2}}, \quad d \equiv \frac{\varrho_{\mathrm{i}}}{\varrho_{\mathrm{e}}}, \quad v \equiv \frac{c_{\mathrm{Ae}}}{c_{\mathrm{Ai}}}$,

where $c_{\mathrm{Ae}}=B_{\mathrm{e}} / \sqrt{\mu \varrho_{\mathrm{e}}}$ and $c_{\mathrm{Ai}}=B_{\mathrm{i}} / \sqrt{\mu \varrho_{\mathrm{i}}}$. Henceforth, we choose and hold fixed

$d=3.89, \quad v=2$,

which gives us the plasma $\beta=0.003$.

\subsection{Linear equations}

Small amplitude perturbations of the above described equilibrium obey the coupled wave equations (e.g., Roberts 2000):

$$
\begin{aligned}
& \varrho_{0}\left(\frac{\partial^{2}}{\partial t^{2}}-c_{\mathrm{A}}^{2} \frac{\partial^{2}}{\partial z^{2}}\right) V_{r}+\frac{\partial^{2} p_{\mathrm{T}}}{\partial r \partial t}=0, \\
& \frac{\partial^{2} V_{z}}{\partial t^{2}}-c_{\mathrm{s}}^{2} \frac{\partial}{\partial z}(\nabla \cdot \boldsymbol{V})=0 \\
& \varrho_{0}\left(\frac{\partial^{2}}{\partial t^{2}}-c_{\mathrm{A}}^{2} \frac{\partial^{2}}{\partial z^{2}}\right) V_{\phi}+\frac{1}{r} \frac{\partial^{2} p_{\mathrm{T}}}{\partial \phi \partial t}=0
\end{aligned}
$$


where $p_{\mathrm{T}}$ is the perturbed total pressure such that

$p_{\mathrm{T}} \equiv p+\frac{1}{\mu} \boldsymbol{B}_{0} \cdot \boldsymbol{B}$

Its time evolution is described by the equation

$\frac{\partial p_{\mathrm{T}}}{\partial t}=\varrho_{0} c_{\mathrm{A}}^{2} \frac{\partial V_{z}}{\partial z}-\varrho_{0} c_{\mathrm{f}}^{2} \nabla \cdot \boldsymbol{V}$

Here $c_{\mathrm{s}}(r)=\sqrt{\gamma p_{0}(r) / \varrho_{0}(r)}$ and $c_{\mathrm{f}}(r)=\sqrt{c_{\mathrm{s}}^{2}+c_{\mathrm{A}}^{2}}$ are the sound and fast speeds, respectively.

Equations (12)-(14) describe fast, slow and torsional (or Alfvén) waves modes perturbing $V_{r}, V_{z}$ and $V_{\phi}$, respectively. In general, these three modes are linearly coupled with each other through the perturbation of the total pressure $p_{\mathrm{T}}$.

\section{Numerical model}

MHD Eqs. (1)-(5) are solved numerically using the FLASH code (Zingale et al. 2002) which is a good tool for numerically solving MHD equations. The high-order Riemann solver of Roe type, implemented in this code, yields accurate results near steep gradients and moving contact discontinuities. The MHD part is implemented with a use of Powell's method (Powell 1999) which allows the divergence of the magnetic field to be kept free of machine-dependent round-off errors. The computer code is formally second-order accurate in space and time.

The plasma equations are solved numerically in an $x-y-z$ Eulerian box with dimensions $(-10 \mathrm{a}, 10 \mathrm{a}) \times(-10 \mathrm{a}, 10 \mathrm{a}) \times$ $(-10 \mathrm{a}, 10 \mathrm{a})$. For most numerical runs 36 (18) blocks were chosen in the $x, y(z)$ directions. The maximum refinement level was specified as 8 . We apply free-boundary conditions at the boundaries of the simulation region. This choice of boundary conditions is a consequence of the extended nature of the real medium. In this way, we assume that MHD waves have no time to travel the entire length of the loop and that they have not reached the ends of the loop, where line-tying in the dense lower atmosphere causes reflection, and so the wave propagates freely as if the structure were open.

\section{Impulsively generated MHD waves}

Consider a coronal plasma which is modeled by Eqs. (6) and (9). Perturbations which are excited in the solar corona are very complex. Here we focus our attention on three separate cases which correspond to impulsively excited waves. Such excitation may be due to a flare or any other sudden release of energy process.

Introducing normalized variables, we shall measure plasma velocities in units of $c_{\mathrm{Ae}}$, spatial variables in $a$ and time in units of the Alfvén transit time $t_{\mathrm{A}}=a / c_{\mathrm{Ae}}$. The normalised unit of time can be considered equivalent to one second.

\subsection{Slow wave}

One of the simplest conceivable models is the case when a slow magnetosonic wave is initially present in the medium. Such a
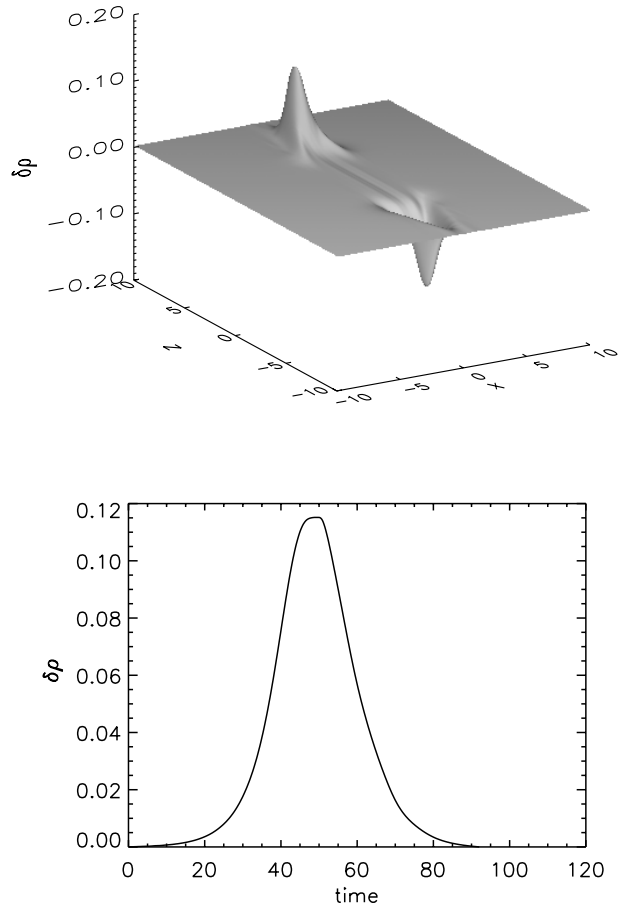

Fig. 1. The spatial profile of the perturbed mass density $\delta \varrho(x, y=0$, $z, t=80)$ (top panel) and the time signature which is obtained by measuring the wave signal in $\delta \varrho$ at $x=y=0, z=4: \delta \varrho$ (bottom panel) in the case of the initially launched pulse of Eq. (17) which essentially corresponds to a slow wave.

wave can be effectively excited by setting the following initial condition:

$V_{z}(r, \phi, z, t=0)=\frac{V_{z 0}}{\cosh ^{2}\left(\frac{r}{a}\right) \cosh ^{2}\left(\frac{z}{a}\right)}, V_{r}=V_{\phi}=0$,

where we choose the pulse amplitude $V_{z 0}=0.005$.

The initial profile of Eq. (17) excites oscillations in the perturbed mass density profile,

$\delta \varrho \equiv \varrho-\varrho_{0}$,

which is shown in the top panel of Fig. 1 at $t=80$. As a result of the initial impact, the region above the initial pulse is compressed while the region $z<0$ is rarified. These profiles are symmetric around the $z$-axis.

The slow wave presented above exhibits time signatures which are made by collecting in time wave signals at the detection point. As the slow pulse does not experience significant dispersive distortion, it preserves the information about the initial pulse and we choose the detection point on the loop axis at $z=4$.

It has been shown by Roberts et al. (1984) that slow waves propagate with a speed which is greater than $c_{\mathrm{ti}}$ and lower than $c_{\mathrm{si}}$. Here, $c_{\mathrm{si}}$ is the sound speed inside the coronal loop and $c_{\mathrm{ti}}$ is the so-called tube speed inside the coronal loop

$\frac{1}{c_{\mathrm{ti}}}=\sqrt{\frac{1}{c_{\mathrm{si}}^{2}}+\frac{1}{c_{\mathrm{Ai}}^{2}}}$. 
So the speed $c_{\mathrm{ti}}$ is sub-sonic and sub-Alfvénic. For the low $\beta$ plasma conditions, $c_{\mathrm{ti}}$ is close to the sound speed inside the loop. It follows from the equilibrium conditions that

$\frac{c_{\mathrm{si}}^{2}}{c_{\mathrm{Ae}}^{2}}=\frac{\gamma}{2} \frac{(\beta+1) v^{2}-d}{d v^{2}}$

For a choice of the equilibrium parameters given by Eq. (11), we have

$\frac{c_{\mathrm{si}}}{c_{\mathrm{Ae}}} \approx 0.077$

Thus the slow wave should reach the detection point $r=0$, $z=4$ at $t \approx 52$, in good agreement with Fig. 1 (bottom panel).

Equations (12) and (13) show that slow and fast magnetoacoustic waves are coupled, while in this case the Alfvén wave decouples from the magnetosonic waves and therefore it is absent from the system. As a consequence of this coupling the fast wave must be present in the system, being driven by the slow magnetosonic wave. However, the fast wave is of the amplitude $10^{-5}$ which is much lower than the slow wave amplitude. As a consequence of this, such a small wave would be hardly detectable in the real system which always contains a noisy background. So this wave is insignificant and therefore it is not shown.

\subsection{Fast sausage wave}

In this case we initially launch a pulse of the form

$V_{r}(r, \phi, z, t=0)=\frac{V_{0 r} r}{\cosh ^{2}\left(\frac{r}{a}\right) \cosh ^{2}\left(\frac{z}{a}\right)}, V_{\phi}=V_{z}=0$.

Here $V_{0 r}=0.005$ is the pulse amplitude. This pulse basically excites fast sausage waves which correspond to symmetric pulsations of the loop, with the central axis of this loop remaining undisturbed. In particular, the radial component of plasma motions disappears at this axis and it changes sign at $r=0$. This wave is unable to propagate for a sufficiently long wavelength as it leaks energy outside the loop. This leakage is represented by the circular wave patterns in the top panel of Fig. 2 which displays the spatial profile of $\delta \varrho$ at $t=9$. This profile corresponds to a fast sausage mode and it is symmetric around the $z$-axis. The Alfvén wave is absent in the system as it decouples from the magnetosonic waves.

Time signatures that are associated with the fast sausage wave are shown in Fig. 2 (bottom panel). Note that the fast sausage wave exhibits short-period oscillations, a scenario which is quite different from the case of the slow wave of Fig. 1 (bottom panel). These several-second oscillations are a result of the fact that sausage modes are more dispersive than a slow wave and the former are leaky for sufficiently long wavelength oscillations. As a consequence of this, only short oscillations are guided along the coronal loop.
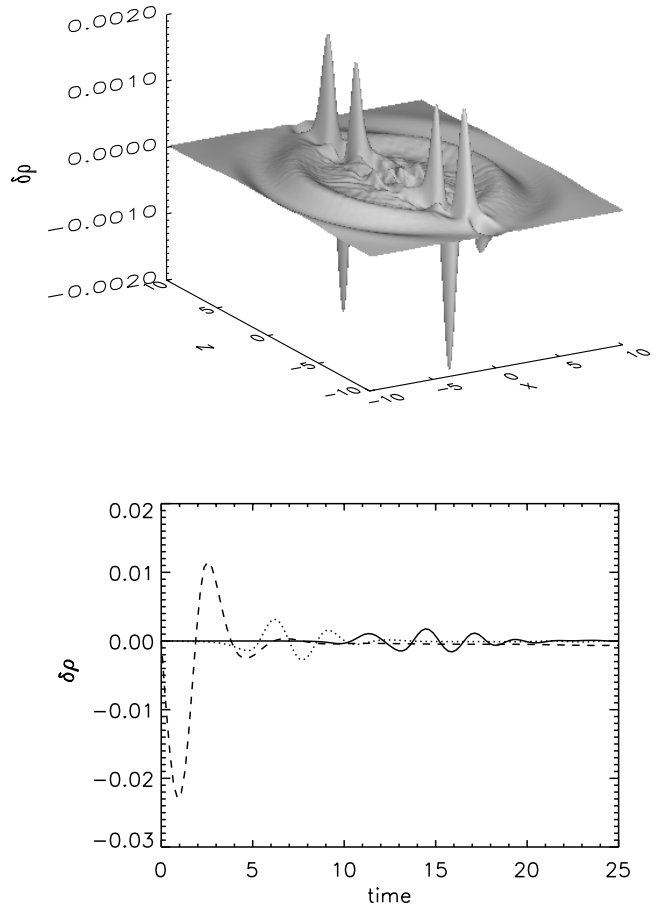

Fig. 2. The spatial profile of $\delta \varrho(x, y=0, z, t=9)$ (top panel) and a time signature which is obtained by measuring the mass density at the detection point $x=y=0, z=0$ (dashed line), $z=4$ (dotted line), and $z=9$ (solid line) (bottom panel) in the case of the initial condition (21) that corresponds to a fast sausage wave.

\subsection{Fast kink wave}

A simple way to excite a fast kink wave in the system is to launch the following pulse:

$V_{x}(x, y, z, t=0)=\frac{V_{x 0}}{\cosh ^{2}\left(\frac{x-x_{0}}{a}\right) \cosh ^{2}\left(\frac{y}{a}\right) \cosh ^{2}\left(\frac{z}{a}\right)}$,

$V_{y}=V_{z}=0$,

where $V_{x 0}=0.005$ is a pulse amplitude and $x_{0}$ its initial position. This pulse excites a packet of waves in which the strongest contribution is from a fast kink wave. A pure kink wave involves lateral displacements of the loop, which maintains a circular cross-section, with the axis of the loop resembling a wriggling snake. We may view this as a global mode of oscillation of the coronal loop, in which the whole loop vibrates. This mode exists for all wavelengths as a trapped oscillation of the loop. Obviously, at the loop center, $r=0$, the radial component of plasma motions generally differs from zero, $V_{r} \neq 0$.

Roberts et al. (1984) show that the kink mode moves at a speed $c_{\mathrm{k}}$ which is determined by both $c_{\mathrm{Ae}}$ and $c_{\mathrm{Ai}}$; the kink mode speed is higher than $c_{\mathrm{Ai}}$ and lower than $c_{\mathrm{Ae}}$. In the long wavelength limit $(\lambda \gg a)$, the phase speed of the principal kink wave is given by the kink speed $c_{\mathrm{k}}$ (Roberts et al. 1984) such that

$c_{\mathrm{k}}=\sqrt{\frac{\varrho_{\mathrm{i}} c_{A i}^{2}+\varrho_{\mathrm{e}} c_{\mathrm{Ae}}^{2}}{\varrho_{\mathrm{i}}+\varrho_{\mathrm{e}}}}$

This speed is the mean Alfvén speed of the medium, intermediate between the Alfvén speed inside the loop and the Alfvén 


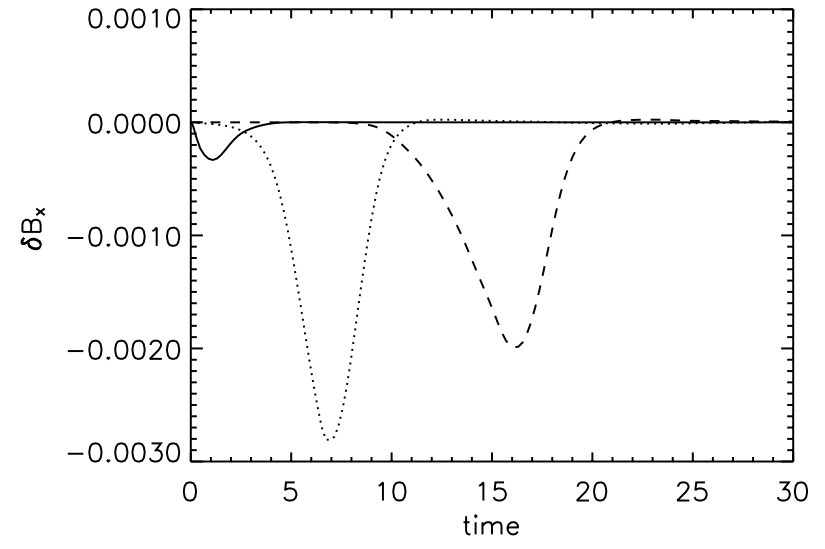

Fig. 3. Time signatures which are obtained by measuring appropriate signals in $\delta B_{x}$ at the detection point $x=y=0, z=0$ (dashed line), $z=4$ (dotted line), and $z=9$ (solid line) for the initial condition (22) with $x_{0}=0$.

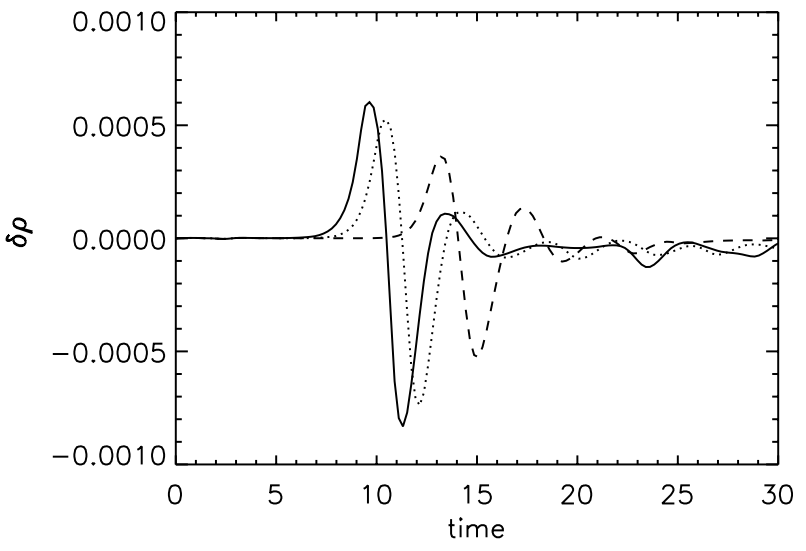

Fig. 4. The time signature obtained by measuring the mass density at the detection point $x=y=0, z=0$ (solid line), $z=4$ (dotted line), and $z=9$ (dashed line) which are generated from the initial condition (22) with $x_{0}=-10$.

speed in the ambient medium. It is interesting that $c_{\mathrm{k}}$ is closer to $c_{\mathrm{Ai}}$, particularly for shorter waves. Consequently, the kink wave reaches the detection point $(r=0, z=4)$ at $t \approx 8$. Figure 3 illustrates this feature of the dispersion relation for fast kink waves. This figure shows a signal in $B_{x}$, as the perturbed mass density profile is of a low magnitude and therefore is not displayed.

Now we discuss the case of the initial pulse of Eq. (22) with $x_{0}=-10$. Such pulse produces complex time signatures. Indeed, Figs. 4 and 5 show time signatures which are made by collecting the perturbed mass density and velocity at three spatial points: (a) $(0,0,0)$ (dashed line); (a) $(0,0,4)$ (dotted line); (a) $(0,0,9)$ (solid line). It is discernible that with a larger distance from the exciter time signatures are more complex.

As the fast and slow waves are coupled we expect a signal to be present in the longitudinal component of velocity, $V_{z}$. Obviously, this signal is of a lower magnitude that the signal in $V_{x}$ (not shown). In this case, the initial condition (22) also excites a signal in $V_{\phi}$ which correspondes to the Alfvén wave (not shown).
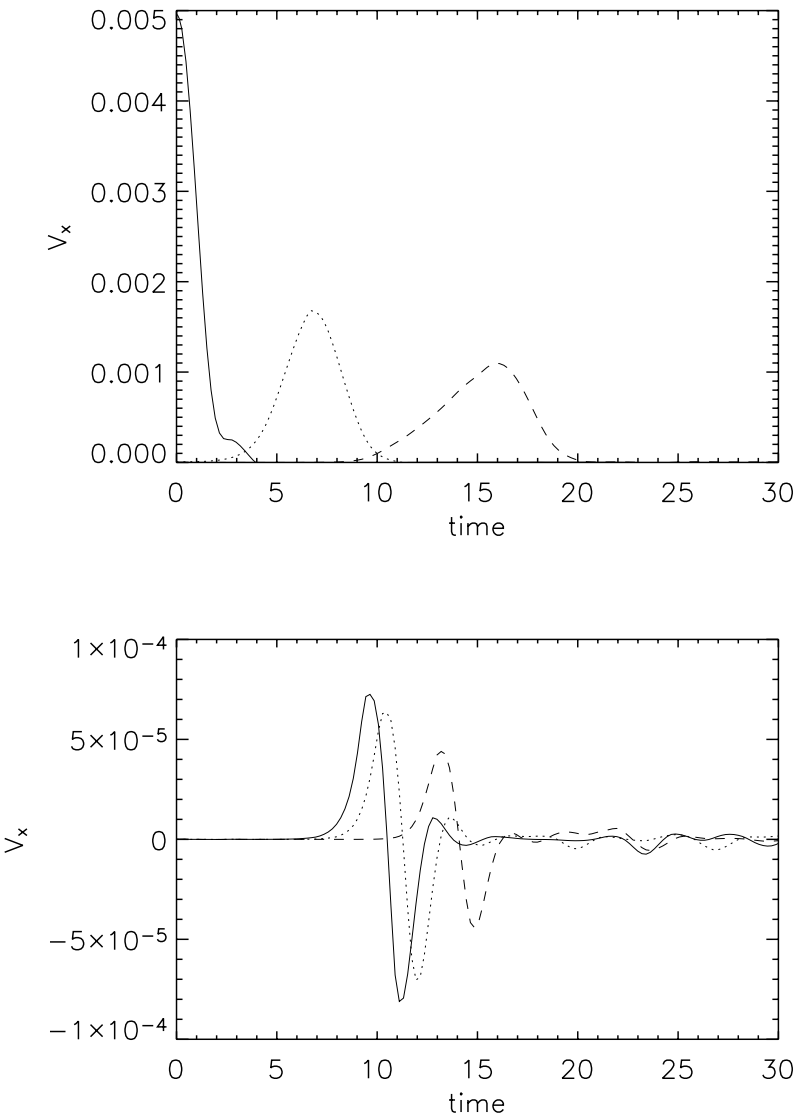

Fig. 5. The time signatures which are obtained by measuring the $x$-component of velocity at the detection point $x=y=0, \quad z=0$ (solid line), $z=4$ (dotted line), and $z=9$ (dashed line). These time signatures are generated from the initial condition (22) with $x_{0}=0$ (top panel) and with $x_{0}=-10$ (bottom panel).

\section{Summary}

We have developed a simple model of the coronal plasma. This model makes no allowance for loop curvature or stratification, nor is there any realistic modeling of the coupling of the coronal plasma to the denser layers of the solar atmosphere. Using this model, we have numerically simulated the response of the coronal plasma to impulsively generated MHD waves. We have adapted the FLASH code (Zingale et al. 2002) which is based on a Godunov-type method (e.g., Murawski 2002). In the Godunov method (Godunov 1959) the interaction of a pair of numerical cells at their interface is assumed to take place through a number of waves (LeVeque 2002). This yields an accurate scheme which avoids non-physical oscillations in the vicinity of shock waves.

The primary result of the study reported here is the demonstration of the importance of the exciting conditions on the propagation of magnetosonic waves. Our results both complement and extend those of earlier published studies, and we have attempted to clarify some of the subtler aspects of the wave theory. By such an approach we might embrace the important physical processes of oscillations studied in geophysics, oceanography, atmospheric, and astrophysical contexts. 
A slow pulse $\left(V_{z}\right)$ is associated with a relatively strong perturbation of the mass density. A fast sausage pulse $\left(V_{r}\right)$ does not lead to a localized perturbation of the mass density in the loop. This is a consequence of the fact that short waves are trapped by the loop while it is leaky to long waves. An infinitely long kink wave can oscillate in a loop but when the initial pulse is located on the loop axis it does not significantly perturb the mass density. On the other hand, an external pulse leads to mass density alterations with complex time signatures.

A slow magnetosonic wave generates a wave profile with a time scale of the order of $\sim 40 \mathrm{~s}$. Time scales which are associated with the fast kink wave are generally lower than for a slow wave: they are in the vicinity of $\sim 12 \mathrm{~s}$. Temporal signatures of a fast sausage wave reveal $\sim 5 \mathrm{~s}$ oscillations in the quasi-periodic phase. These scales are in general agreement with the results of recent coronal wave observations (Williams et al. 2002).

Last but not least we would like to stress the importance of the inclusion of an impulse external to the loop in our modelling of the fast kink wave. This is a useful perspective on loop wave dynamics, since it brings to mind the flare-induced oscillations of TRACE loops observed by Aschwanden et al. (1999), Nakariakov \& Ofman (2001) and Schrijver et al. (2002).

Acknowledgements. The authors express their cordial thanks to Drs. Valery Nakariakov and Markus Aschwanden for stimulating discussions and to the referee, Dr. D. R. Williams for his constructive comments. This work was financially supported by a grant from the State Committee for Scientific Research Republic of Poland, KBN grant No. 2 PO3D 016 25. The software used in this work was in part developed by the DOE-supported ASCI/Alliance Center for Astrophysical Thermonuclear Flashes at the University of Chicago.

\section{References}

Aschwanden, M. 2003, Proc. NATO Workshop, Budapest

Aschwanden, M., Fletcher, L., Schrijver, C. J., \& Alexander, D. 1999, ApJ, 520, 880

Edwin, P. M., \& Roberts, B. 1983, Sol. Phys., 88, 179

Godunov, S. K. 1959, Mat. Sb., 47, 271

Handy, B. N., Acton, L. W., Kankelborg, C. C., et al. 1999, Sol. Phys., 187,229

LeVeque, R. J. 2002, Finite-volume methods for hyperbolic problems (Cambridge)

Murawski, K. 2002, Analytical and numerical methods for wave propagation in fluids (Singapore: World Scientific)

Murawski, K., \& Roberts, B. 1993, Sol. Phys., 144, 255

Murawski, K., \& Roberts, B. 1994, Sol. Phys., 151, 305

Murawski, K., Aschwanden, M. J., \& Smith, J. M. 1998, Sol. Phys., 179,313

Nakariakov, V. M. 2003, in The Dynamic Sun, ed. B. Dwivedi (CUP)

Nakariakov, V. M., \& Ofman, L. 2001, A\&A, 372, L53

Nakariakov, V. M., Ofman, L., DeLuca, E. E., et al. 1999, Science, 285,862

Nakariakov, V. M., Arber, T. D., Ault, C. E., et al. 2004, MNRAS, 349, 705

Phillips, K. J. H., Read, P. D., Gallagher, P. T., et al. 2000, Sol. Phys., 193, 259

Powell, K. 1999, J. Comp. Phys., 154, 284

Roberts, B. 2000, Sol. Phys., 193, 139

Roberts, B. 2003, Proc. Workshop, Prague

Roberts, B., Edwin, P. M., \& Benz, A. O. 1984, ApJ, 279, 857

Schrijver, C. J. Aschwanden, M. J., \& Title, A. M. 2002, Sol. Phys., 206, 69

Wilhelm, K., Curdt, W., Marsch, E., et al., Sol. Phys., 162, 189

Williams, D. R., Mathioudakis, M., Gallagher, P. T., et al. 2002, MNRAS, 336, 747

Zingale, M., Dursi, L. J., ZuHone, J., et al. 2002, ApJS, 143, 539 